\title{
Ore and Erdős type conditions for long cycles in balanced bipartite graphs
}

\author{
Janusz Adamus $1 \|^{\dagger}$ and Lech Adamus $\|^{\#}$ \\ ${ }^{1}$ Department of Mathematics, The University of Western Ontario, London, Ontario, N6A 5B7 Canada and Institute \\ of Mathematics, Jagiellonian University, ul. Łojasiewicza 6, 30-348 Kraków, Poland \\ ${ }^{2}$ Faculty of Applied Mathematics, AGH University of Science and Technology, Al. Mickiewicza 30, 30-059 Kraków, \\ Poland and LRI, Bât. 490, Université Paris-Sud, 91405 Orsay Cedex, France
}

received February 12, 2009, revised June 15, 2009, accepted June 16, 2009.

We conjecture Ore and Erdős type criteria for a balanced bipartite graph of order $2 n$ to contain a long cycle $C_{2 n-2 k}$, where $0 \leq k<n / 2$. For $k=0$, these are the classical hamiltonicity criteria of Moon and Moser. The main two results of the paper assert that our conjectures hold for $k=1$ as well.

Keywords: bipartite graph, cycle, long cycle, hamiltonicity, degree sum

\section{Introduction}

One of the classical problems of graph theory is the study of sufficient conditions for a graph to contain a Hamilton cycle. In this paper we are primarily interested in two types of such conditions. Namely, the ones that put constraints on degree sums of pairs of non-adjacent vertices, and those that combine bounds on the size of a graph with bounds on its minimal degree. The first approach is due to Ore (see Section 2 for notation):

Theorem 1.1 (Ore, [12]). Let $G$ be a graph of order $n \geq 3$, in which

$$
d_{G}(x)+d_{G}(y) \geq n
$$

for every pair of non-adjacent vertices $x$ and $y$. Then $G$ contains a Hamilton cycle.

It follows immediately from Ore's theorem that the minimal size of a graph of order $n \geq 3$ that guarantees hamiltonicity is $\left(\begin{array}{c}n-1 \\ 2\end{array}\right)+2$. Erdős generalized this condition by adding a bound on the minimal degree of a graph:

\footnotetext{
${ }^{\dagger}$ Corresponding author.

¥The second author’s research was partially supported by the AGH University of Science and Technology grant No. 11.420.04 and by the Polish Ministry of Science doctoral grant No. 0102/M03/2007/32.

1365-8050 @ 2009 Discrete Mathematics and Theoretical Computer Science (DMTCS), Nancy, France
} 
Theorem 1.2 (Erdős, [9]). Let $G$ be a graph of order $n \geq 3$ and minimal degree $\delta(G) \geq r$, where $1 \leq r<n / 2$. Then $G$ contains a Hamilton cycle, provided

$$
\|G\|>\max \left\{\left(\begin{array}{c}
n-r \\
2
\end{array}\right)+r^{2},\left(\begin{array}{c}
n-\left\lfloor\frac{n-1}{2}\right\rfloor \\
2
\end{array}\right)+\left\lfloor\frac{n-1}{2}\right\rfloor^{2}\right\} .
$$

The above conditions can, of course, be significantly strengthened in case of a balanced bipartite graph. The following two theorems are bipartite counterparts of Ore and Erdós criteria, respectively.

Theorem 1.3 (Moon and Moser, [11]). Let $G$ be a bipartite graph of order $2 n$, with colour classes $X$ and $Y$, where $|X|=|Y|=n \geq 2$. Suppose that $d_{G}(x)+d_{G}(y) \geq n+1$ for every pair of non-adjacent vertices $x \in X$ and $y \in Y$. Then $G$ contains a Hamilton cycle.

Theorem 1.4 (Moon and Moser, [11]). Let $G$ be a bipartite graph of order $2 n$, with colour classes $X$ and $Y,|X|=|Y|=n \geq 2$, and minimal degree $\delta(G) \geq r, 1 \leq r \leq n / 2$. Then $G$ contains a Hamilton cycle, provided $\|G\|>n(n-r)+r^{2}$.

Our goal is to generalize the above criteria to long cycles, that is, cycles of length $2 n-2 k$, where $0 \leq k<n / 2$. We state the following two conjectures, that include Theorems 1.3 and 1.4 as special cases $(k=0)$.

Conjecture A. Let $G$ be a 2-connected balanced bipartite graph of order $2 n$, with colour classes $X$ and $Y,|X|=|Y|=n \geq 5$, and let $k<n / 2$ be a non-negative integer. If

$$
d_{G}(x)+d_{G}(y) \geq n-k+1
$$

for every pair of non-adjacent $x \in X$ and $y \in Y$, then $G$ contains a cycle of length $2 n-2 k$.

Conjecture B. Let $G$ be a balanced bipartite graph of order $2 n$ and minimal degree $\delta(G) \geq r \geq 1$, where $n \geq 2 k+2 r$ and $k \in \mathbb{Z}$. If

$$
\|G\|>n(n-k-r)+r(k+r)
$$

then $G$ contains a cycle of length $2 n-2 k$.

The main two results of this paper, Theorems A and B (Section 3), assert that our conjectures hold true for $k=1$. We believe the conjectures to be significantly harder in case $k \geq 2$.

It should be mentioned here that analogous generalizations to long cycles of Ore's and Erdős's theorems have been studied in ordinary graphs. Woodall [14, Thm. 11] gives a complete list of Erdôs type conditions for a graph of order $n$ to contain a cycle of length $n-k$ for any $0 \leq k \leq \frac{n-3}{2}$. The Ore type criterion is conjectured in [1], and follows from a result of Linial [10] in case $k \leq 1$.

Remark 1.5. Both the degree sum condition of Conjecture A and the bound on the size of Conjecture B are sharp, as can be seen in Example 1.6 below. It is also necessary to assume 2-connectedness in Conjecture A (Example 1.7). Finally, a quick look at $C_{6}$ and $C_{8}$ shows that Conjecture A would fail for $n<5$. 
Example 1.6. Let $G_{1}$ be a balanced bipartite graph, with colour classes $X$ and $Y,|X|=|Y|=n$, where $X=A \cup B, Y=C \cup D,|A|=k+r,|B|=n-k-r,|C|=r$, and $|D|=n-r$. Moreover, assume that $N_{G_{1}}(x)=C$ for all $x \in A$, and $N_{G_{1}}(x)=Y$ for all $x \in B$. Then $d_{G_{1}}(x)+d_{G_{1}}(y)=n-k$ for every pair $x \in A$ and $y \in D$, and, in general, $d_{G_{1}}(x)+d_{G_{1}}(y) \geq n-k$ for every pair of $x \in X$ and $y \in Y$. If $n \geq 2 k+2 r$, then $\delta\left(G_{1}\right)=r \geq 1$ and $\left\|G_{1}\right\|=n(n-k-r)+r(k+r)$, but $G_{1}$ does not contain a cycle of length $2 n-2 k$.

Example 1.7. Let $G_{2}=(X, Y ; E)$ be a balanced bipartite graph obtained from the disjoint union of $H_{1}=K_{\lfloor n / 2\rfloor,\lfloor n / 2\rfloor}$ and $H_{2}=K_{\lceil n / 2\rceil,\lceil n / 2\rceil}$ by adding a single edge joining a vertex of $H_{1}$ with a vertex of $H_{2}$. Then $d_{G_{2}}(x)+d_{G_{2}}(y) \geq n$ for every pair of non-adjacent vertices $x \in X$ and $y \in Y$, nonetheless $G_{2}$ contains no cycle of length $2 n-2$. In fact, $G_{2}$ contains no long cycle whatsoever.

The next section contains the inventory of basic definitions and results used throughout the paper. In Section 3 we state our main results, Theorems A and B, and their consequences. In particular, by combining Theorems A and B, we obtain a complete Erdós type characterisation of balanced bipartite graphs that do not contain cycles of length $2 n-2$ (Theorem 3.6). The last two sections are devoted to proofs of the two main results.

\section{Notation and tools}

All graphs considered are undirected, have no loops and no multiple edges. Given a graph $G$, we denote by $\|G\|$ the size (i.e., number of edges) of $G$, and by $V(G)$ the vertex set of $G$. A bipartite graph is often denoted by $G=(X, Y ; E)$, where $X$ and $Y$ are the two colour classes of $G$, and $E=E(G)$ is the edge set of $G$. When $|X|=|Y|$, we say that $G$ is balanced. Given a vertex $x \in V(G), N_{G}(x)$ denotes the set of vertices adjacent to $x$ in $G, d_{G}(x)$ the degree of $x$ in $G$ (i.e., $\left.d_{G}(x)=\left|N_{G}(x)\right|\right)$, and $\delta(G)$ the minimal vertex degree in $G$. If $L \subset V(G)$ is a vertex subset of $G$, then $G-L$ denotes the subgraph of $G$ induced by $V(G) \backslash L$, and $N_{G}(L)$ is the set of neighbours of all the vertices in $L$. Given distinct vertices $x$ and $y$ of $G$, an $x-y$ path is a path in $G$ with endvertices $x$ and $y$. We denote by $C_{l}$ a cycle of length $l$, and by $K_{n, n}$ a complete balanced bipartite graph of order $2 n$. Finally, recall that a graph is called 2-connected if the removal of any single vertex does not disconnect $G$.

In this section we have gathered results used in the proofs of Theorems A and B. First of all, we recall two hamiltonicity criteria obtained by Moon and Moser [11].

Theorem 2.1 (Moon and Moser, [11]). Let $G$ be a balanced bipartite graph of order $2 n \geq 4$, with $\delta(G) \geq \frac{n+1}{2}$. Then $G$ contains a Hamilton cycle.

Theorem 2.2 (Moon and Moser, [11]). Let $G=(X, Y ; E)$ be a balanced bipartite graph of order $2 n$, and let $S_{m}=\left\{x \in X: d_{G}(x) \leq m\right\}, T_{m}=\left\{y \in Y: d_{G}(y) \leq m\right\}$ for $m \in \mathbb{Z}$. If, for every $1 \leq m \leq n / 2$, the sets $S_{m}$ and $T_{m}$ are of cardinalities less than $m$, then $G$ is hamiltonian.

We shall need the following strengthening of Theorem 1.4

Theorem 2.3 (Wojda and Woźniak, [13]). Let $G(n, r)$ denote a bipartite graph with colour classes $X=$ $P \cup Q$ and $Y=R \cup S$ such that $|P|=|R|=r,|Q|=|S|=n-r, N_{G(n, r)}(x)=R$ for all $x \in P$, and $N_{G(n, r)}(x)=Y$ for all $x \in Q$. Let $G$ be a balanced bipartite graph of order $2 n \geq 4$, minimal degree $\delta(G) \geq r \geq 1$, and size $\|G\| \geq n(n-r)+r^{2}$. Then $G$ contains a Hamilton cycle, else $r \leq n / 2$ and $G$ is isomorphic to $G(n, r)$. 
A bipartite graph of order $2 n$ is called bipancyclic if it contains cycles of lengths $2 k$ for all $2 \leq k \leq n$.

Theorem 2.4 (Bagga and Varma, [5]). Let $G=(X, Y ; E)$ be a balanced bipartite graph of order $2 n \geq 8$. If $d_{G}(x)+d_{G}(y) \geq n+1$ for every pair of non-adjacent vertices $x \in X$ and $y \in Y$, then $G$ is bipancyclic.

Theorem 2.5 (Entringer and Schmeichel, [8]). Let $G$ be a hamiltonian bipartite graph of order $2 n \geq 8$. If $\|G\|>n^{2} / 2$, then $G$ is bipancyclic.

We will also need to know the cycle structure of an $n / 2$-regular hamiltonian bipartite graph $G$ of order $2 n$. Notice that then $\|G\|=n^{2} / 2$, so the above theorem does not apply. We then have:

Theorem 2.6 (J. Adamus, [2]). Let $G$ be an n/2-regular hamiltonian bipartite graph of order $2 n$. Then $G$ contains a cycle $C$ of length $2 n-2$. Moreover, if $C$ can be chosen to omit a pair of adjacent vertices, then $G$ is bipancyclic.

Given a balanced bipartite graph $G=(X, Y ; E)$, one defines a $k$-biclosure $B C l_{k}(G)$ of $G$ as the graph obtained from $G$ by succesively joining pairs of non-adjacent vertices $x \in X$ and $y \in Y$, with degree sum of at least $k$, until no such pair remains. Closely related to this construction is the notion of $k$-bistability: A property $\mathcal{P}$ defined on all balanced bipartite graphs of order $2 n$ is called $k$-bistable when, whenever $G+x y$ has the property $\mathcal{P}$ and $d_{G}(x)+d_{G}(y) \geq k$, then $G$ itself has the property $\mathcal{P}$.

Theorem 2.7 (Bondy and Chvátal, [7]). A balanced bipartite graph $G$ of order $2 n$ is hamiltonian if and only if its $(n+1)$-biclosure $B C l_{n+1}(G)$ is so.

Theorem 2.8 (Amar, Favaron, Mago and Ordaz, [4]). The property of containing a cycle of length $2 n-2$ is $(n+2)$-bistable on balanced bipartite graphs of order $2 n$.

\section{Long cycles in balanced bipartite graphs}

Suppose we want to know whether a balanced bipartite graph $G=(X, Y ; E)$ has the property of containing a long cycle $C_{2 n-2 k}$ for some $0 \leq k<n / 2$. Given Theorem 1.3 of Moon and Moser, a natural question arises: Can one impose such a property by decreasing the bound on the degree sum of nonadjacent vertices by $k$ ? We believe the answer to this question be positive (Conjecture A). As shown in Example 1.6, any lower bound on the degree sum of non-adjacent vertices $x \in X$ and $y \in Y$ which ensures $C_{2 n-2 k} \subset G$ is at least $n-k+1$. On the other hand, decreasing the bound below $n+1$ imposes additional assumptions on the graph. Interestingly enough, without the 2-connectedness constraint the graph could contain no long cycles at all (see Example 1.7). The following result gives a positive answer to the above question in case $k=1$.

Theorem A. Let $G=(X, Y ; E)$ be a 2-connected balanced bipartite graph of order $2 n \geq 4$, such that $d_{G}(x)+d_{G}(y) \geq n$ for every pair of non-adjacent vertices $x \in X$ and $y \in Y$. Then $G$ contains an even cycle of length at least $2 n-2$.

We postpone the proof of the theorem to Section 4 Right now we will show that Theorem A implies Conjecture A for $k=1$.

Corollary 3.1. Conjecture A holds for $k=1$. 
Proof: Let $G=(X, Y ; E)$ be a balanced bipartite graph of order $2 n$ that satisfies the assumptions of Conjecture A. By Theorem A above, $G$ contains an even cycle of length at least $2 n-2$, so without loss of generality one may assume that $G$ is hamiltonian.

Let $x \in X$, say, be a vertex of minimal degree $\delta(G)$ in $G$. Then $Y$ contains precisely $n-\delta(G)$ vertices non-adjacent to $x$, each of degree at least $n-\delta(G)$ (as $d_{G}(x)+d_{G}(y) \geq n$ for $\left.x y \notin E\right)$. Counting the edges incident with $Y$, we get

$$
\|G\| \geq(n-\delta(G)) \cdot(n-\delta(G))+\delta(G) \cdot \delta(G) .
$$

Observe that $(n-\delta(G))^{2}+\delta(G)^{2}>n^{2} / 2$ iff $\delta(G) \neq n / 2$. Hence $\|G\|>n^{2} / 2$, provided $\delta(G) \neq n / 2$, and thus $G$ contains $C_{2 n-2}$, by Theorem 2.5. If, in turn, $\delta(G)=n / 2$, then the result follows from Theorem 2.6

Let us now turn to Erdős type criteria. In [3], the second author conjectured the following sufficient condition for a balanced bipartite graph to contain a long cycle $C_{2 n-2 k}$ (proved in [3] under considerably stronger assumptions).

Conjecture 3.2 (L. Adamus, [3]). Let $G$ be a balanced bipartite graph of order $2 n$, where $n \geq 2 k+2$, $k \in \mathbb{Z}$. If $\|G\|>n(n-k-1)+k+1$, then $G$ contains a cycle of length $2 n-2 k$.

Notice that both assumptions of the conjecture are weakest possible, as shown by the following two examples.

Example 3.3. Consider a graph $G_{1}$ of Example 1.6 with $r=1$. This graph has precisely $n(n-k-1)+$ $k+1$ edges, and it contains no cycle of length greater than $2 n-2 k-2$.

Example 3.4. Let $G_{3}=(X, Y ; E)$ be a balanced bipartite graph, with colour classes of the form $X=$ $A \cup B, Y=C \cup D$, where $|A|=|D|=k+1,|B|=|C|=n-k-1$. Fix a vertex $y_{0}$ in $C$, and let $N_{G_{3}}(x)=C$ for all $x \in A$, and $N_{G_{3}}(x)=D \cup\left\{y_{0}\right\}$ for all $x \in B$. Then $\left\|G_{3}\right\|>n(n-k-1)+k+1$ for $k+3 \leq n \leq 2 k+1$, yet $G_{3}$ contains no cycle of length greater than $2 n-2 k-2$. Hence the necessity of the assumption $n \geq 2 k+2$.

Interestingly, a similar graph was recently shown in [6] to be a counterexample to Győri's conjecture on $C_{2 l}$-free bipartite graphs.

In light of Example 3.3 above, we ask: By how much can we decrease the lower bound on the size of a given graph $G$ ensuring the existence of a cycle of length $2 m-2 k$, knowing that the minimal degree of $G$ is greater than 1 ? We address this question in Conjecture B. Certain special cases of Conjecture B are known true: $k=0$ is Theorem 1.4, $k=r=1$ is done in [3]. The following theorem (proved in Section 5 below) shows that the conjecture also holds for $k=1$ and arbitrary $r$.

Theorem B. Let $G=(X, Y ; E)$ be a balanced bipartite graph of order $2 n$ and minimal degree $\delta(G) \geq$ $r \geq 1$, where $n \geq 4$ and $n \geq 2 r+1$. Let

$$
g(n, r)=n(n-1-r)+r(1+r)+1
$$

Then $G$ contains a cycle of length $2 n-2$, provided $\|G\| \geq g(n, r)$.

Notice that Theorems 2.1 and 1.4 can be put together as follows: 
Theorem 3.5. Let $G$ be a balanced bipartite graph of order $2 n \geq 4$, with minimal degree $\delta(G) \geq r$. Then $G$ contains a Hamilton cycle, provided

(1) $n \leq 2 r-1$ or

(2) $n \geq 2 r$ and $\|G\|>n(n-r)+r^{2}$.

Along the same lines, we combine Theorem 2.4 and Theorems A and B to prove the following criterion for cycles of length $2 n-2$.

Theorem 3.6. Let $G=(X, Y ; E)$ be a balanced bipartite graph of order $2 n \geq 8$, with minimal degree $\delta(G) \geq r \geq 1$. Then $G$ contains a cycle of length $2 n-2$, provided

(1) $n \leq 2 r-1$ or

(2) $n=2 r$ and $\|G\| \geq 2 r^{2}+r+1$ or

(3) $n \geq 2 r+1$ and $\|G\| \geq n(n-1-r)+r(1+r)+1$.

Remark 3.7. The lower bounds of conditions (2) and (3) are sharp: For an extremal graph for (2), consider the graph $G_{3}$ from Example 3.4 with $k+1=r$; for $(3)$, consider $G_{1}$ from Example 1.6 with $k=1$.

\section{Proof of Theorem 3.6:}

(1) Since $n \leq 2 r-1$ iff $r \geq(n+1) / 2$, then the degree sum is greater than or equal to $n+1$ for every pair of vertices in $G$ (in particular, for non-adjacent ones). By Theorem 2.4, $G$ is then bipancyclic.

(2) The bound on the size of $G$ together with $\delta(G) \geq r=n / 2$ force 2 -connectedness. Also, the degree sum is at least $2 r=n$ for every pair of vertices in $G$. Hence, by Corollary 3.1 . $G$ contains $C_{2 n-2}$.

(3) This is Theorem B.

\section{Proof of Theorem A}

As 2-connectedness of a graph $G$ implies $\delta(G) \geq 2$, the assertion of the theorem holds true for $n \leq 3$, by Theorem 2.1. Suppose then there exists $n \geq 4$ for which the assertion fails. Let $G=(X, Y ; E)$ be a maximal 2-connected balanced bipartite graph of order $2 n$, in which $d_{G}(x)+d_{G}(y) \geq n$ for all non-adjacent $x \in X, y \in Y$, without a cycle of length at least $2 n-2$. By maximality of $G, G+x y$ contains a cycle of length at least $2 n-2$, and hence $G$ contains an $x-y$ path of length $2 n-3$ or $2 n-1$ for every pair of non-adjacent $x \in X, y \in Y$.

We shall show first that $G$ contains a Hamilton path. Suppose not. Let $x \in X, y \in Y$ be non-adjacent vertices and let $P$ be an $x-y$ path in $G$ of length $2 n-3$; say, $P=u_{1} v_{1} u_{2} v_{2} \ldots u_{n-1} v_{n-1}$, where $X=\left\{u_{1}, \ldots, u_{n}\right\}, Y=\left\{v_{1}, \ldots, v_{n}\right\}, u_{1}=x$ and $v_{n-1}=y$. Put $I_{P}=\left\{1 \leq i \leq n-1 \mid u_{1} v_{i} \in E\right\}$ and $J_{P}=\left\{1 \leq i \leq n-1 \mid u_{i} v_{n-1} \in E\right\}$. Then $I_{P} \cap J_{P}=\emptyset$, for if $i_{0} \in I_{P} \cap J_{P}$, then $G$ contains a cycle $u_{1} v_{i_{0}} u_{i_{0}+1} \ldots v_{n-1} u_{i_{0}} v_{i_{0}-1} \ldots v_{1} u_{1}$ of length $2 n-2$; a contradiction. 
As $\left|I_{P}\right|=d_{G[V(P)]}(x)$ and $\left|J_{P}\right|=d_{G[V(P)]}(y)$, we obtain

$$
d_{G[V(P)]}(x)+d_{G[V(P)]}(y)=\left|I_{P}\right|+\left|J_{P}\right|=\left|I_{P} \cup J_{P}\right| \leq n-1,
$$

where $G[V(P)]$ denotes the subgraph of $G$ induced by the vertex set of $P$. This shows that at least one of the vertices $u_{1}$ and $v_{n-1}$ has a neighbour among the remaining vertices $u_{n}, v_{n}$ of $G-P$; say, $v_{n-1} u_{n} \in E$. Notice that then $u_{n} v_{n} \notin E$, for otherwise $u_{1} \ldots v_{n-1} u_{n} v_{n} u_{1}$ would be a Hamilton path. Similarly, $u_{1} v_{n} \notin E$. Hence, in particular, $I_{P}$ contains indices of all the neighbours of $u_{1}$ in $G$, so $\left|I_{P}\right|=d_{G}\left(u_{1}\right)$. Let now $K_{P}=\left\{1 \leq i \leq n-1 \mid u_{i} v_{n} \in E\right\}$. Then $\left|K_{P}\right|=d_{G}\left(v_{n}\right)$, and as $d_{G}\left(u_{1}\right)+d_{G}\left(v_{n}\right) \geq n$, it follows that there exists $i_{0} \in I_{P} \cap K_{P}$. Then $v_{n} u_{i_{0}} v_{i_{0}-1} \ldots u_{1} v_{i_{0}} u_{i_{0}+1} \ldots v_{n-1} u_{n}$ is a Hamilton path in $G$; a contradiction.

Let now $x \in X$ and $y \in Y$ be a pair of non-adjacent vertices such that $G$ contains a Hamilton $x-y$ path $P$; say, $P=u_{1} v_{1} \ldots u_{n} v_{n}$, where $X=\left\{u_{1}, \ldots, u_{n}\right\}, Y=\left\{v_{1}, \ldots, v_{n}\right\}, x=u_{1}$ and $y=v_{n}$. Put $I_{G}=\left\{1 \leq i \leq n \mid u_{1} v_{i} \in E\right\}$ and $J_{G}=\left\{1 \leq i \leq n \mid u_{i} v_{n} \in E\right\}$. Then $\left|I_{G}\right|=d_{G}(x),\left|J_{G}\right|=d_{G}(y)$ and $I_{G} \cap J_{G}=\emptyset$, for if $i_{0} \in I_{G} \cap J_{G}$, then $u_{1} v_{i_{0}} u_{i_{0}+1} \ldots v_{n} u_{i_{0}} v_{i_{0}-1} \ldots v_{1} u_{1}$ is a Hamilton cycle in $G$. Hence

$$
n \geq\left|I_{G} \cup J_{G}\right|=\left|I_{G}\right|+\left|J_{G}\right|=d_{G}(x)+d_{G}(y) \geq n,
$$

so that, for every $1 \leq i \leq n$,

$$
\text { either } u_{i} \in N_{G}(y) \text { or else } v_{i} \in N_{G}(x) \text {. }
$$

Let $d=d_{G}(y)$. Denote by $x_{1}, \ldots, x_{d}$ those of the vertices $u_{1}, \ldots, u_{n}$ that are adjacent to $y$, ordered according to the orientation of $P$ (from $x$ to $y$ ). Let $y_{1}, \ldots, y_{d}$ be the vertices of $Y$ that lie on $P$ next to the respective $x_{1}, \ldots, x_{d}$; then $y_{d}=y$.

Observe that if $x_{1}=u_{i}$ with $i<n-d+1$, then there exists $1 \leq j \leq d-1$ such that $y_{j}=v_{l}$, where $u_{l+1} \notin N_{G}(y)$. Then $v_{l+1} \in N_{G}(x)$ and we obtain a cycle $u_{1} v_{l+1} u_{l+2} \ldots v_{n} u_{l} v_{l-1} \ldots v_{1} u_{1}$ of length $2 n-2$ in $G$; a contradiction.

Therefore $x_{1}=u_{n-d+1}$, and hence $N_{G}(y)$ coincides with the set $\left\{u_{n-d+1}, \ldots, u_{n}\right\}$, call it $U$. Then $\left\{y_{1}, \ldots, y_{d}\right\}$ coincides with $V:=\left\{v_{n-d+1}, \ldots, v_{n}\right\}$, and by $(\star), N_{G}(x)=Y \backslash V$.

Suppose now that, for every $v \in V, N_{G}(v) \subset U$. Then, for all $u \in X \backslash U$ and $v \in V, u$ and $v$ are non-adjacent, hence $N_{G}(u) \subset Y \backslash V$. Consequently, $d_{G}\left(u_{i}\right) \leq n-d(i \leq n-d)$, and $d_{G}\left(v_{j}\right) \leq d$ $(j \geq n-d+1)$. But $u_{i}$ and $v_{j}$ being non-adjacent, we also have $d_{G}\left(u_{i}\right)+d_{G}\left(v_{j}\right) \geq n$, which implies that $d_{G}\left(u_{i}\right)=n-d$ and $d_{G}\left(v_{j}\right)=d$, and hence

$$
N_{G}\left(u_{i}\right)=Y \backslash V \text { and } N_{G}\left(v_{j}\right)=U \text { for all } i \leq n-d, j \geq n-d+1 .
$$

Thus $G$ contains a complete bipartite graph $K_{d, d}$ spanned on the vertices of $U$ and $V$, and a complete bipartite $K_{n-d, n-d}$ spanned on $X \backslash U$ and $Y \backslash V$.

Now, $G$ being 2-connected, it must contain two independent edges $u_{i_{1}} v_{j_{1}}$ and $u_{i_{2}} v_{j_{2}}$ for some $i_{1}, i_{2} \geq$ $n-d+1$ and $j_{1}, j_{2} \leq n-d$. One immediately verifies that such a graph contains a cycle of length $2 n-2$, again contradicting the choice of $G$.

We can therefore conclude that there exists a vertex $v_{j}$, with $n-d+1 \leq j \leq n-1$, adjacent to a $u_{i}$, where $i \leq n-d$. Then $u_{1} v_{i} \ldots u_{j} v_{n} u_{n} \ldots v_{j} u_{i} v_{i-1} \ldots v_{1} u_{1}$ is a Hamilton cycle in $G$. This contradiction completes the proof of the theorem. 


\section{Proof of Theorem B}

Throughout this section we will frequently refer to the exceptional graph $G(n, r)$ of Theorem 2.3 Recall that by $G(n, r)$ we denote a balanced bipartite graph of order $2 n$, with colour classes $X=P \cup Q$ and $Y=R \cup S$, where $|P|=|R|=r,|Q|=|S|=n-r, N_{G(n, r)}(x)=R$ for all $x \in P$, and $N_{G(n, r)}(x)=Y$ for all $x \in Q$.

Let, as before, $g(n, r)=n(n-1-r)+r(1+r)+1$. We shall first show the following lemma.

Lemma 5.1. Let $G=(X, Y ; E)$ be a balanced bipartite graph of order $2 n$ and minimal degree $\delta(G) \geq$ $r \geq 1$, where $n \geq 4$ and $n \geq 2 r+1$. Let $\|G\| \geq g(n, r)$, and assume there exists a pair of vertices $x \in X$ and $y \in Y$ such that $d_{G}(x)+d_{G}(y) \leq n$ and $\delta(G-\{x, y\}) \geq r$. Then $G$ contains a cycle of length $2 n-2$.

Proof: Suppose $G$ contains no cycle of length $2 n-2$. Then $G-\{x, y\}$ contains no such cycle either, and as $\delta(G-\{x, y\}) \geq r$, Theorem 2.3 implies that

$$
\|G-\{x, y\}\| \leq(n-1)(n-1-r)+r^{2}=n^{2}-2 n-n r+r^{2}+r+1 .
$$

On the other hand,

$$
\|G-\{x, y\}\| \geq g(n, r)-\left(d_{G}(x)+d_{G}(y)\right) \geq n^{2}-2 n-n r+r^{2}+r+1 .
$$

Hence $d_{G}(x)+d_{G}(y)=n$, the vertices $x$ and $y$ are non-adjacent, $G-\{x, y\}$ equals $G(n-1, r)$, and $r \leq(n-1) / 2$. Without loss of generality, we may assume that $x$ belongs to the colour class of $G$ containing $P \cup Q$ of $G(n-1, r)$.

Now, either $d_{G}(x) \geq r+1$ or $d_{G}(x)=r$. In the first case, $x$ must have at least two neighbours in $S$ or else at least one neighbour in both $S$ and $R$. One easily verifies that then $G$ contains a cycle of length $2 n-2$, omitting $y$ and a single vertex of $P$; a contradiction.

If, in turn, $d_{G}(x)=r$, then $d_{G}(y)=n-r$ and $y$ must have neighbours in both $P$ and $Q$, since $r \leq(n-1) / 2<n / 2$. Consequently, $G$ contains a cycle of length $2 n-2$, omitting $x$ and a vertex of $S$, which again contradicts the choice of $G$.

We are now in position to prove Theorem B.

For a proof by contradiction, consider a graph $G$ satisfying the assumptions of Theorem B, that does not contain a cycle of length $2 n-2$. Observe first that $\|G\|>n^{2} / 2$. Indeed, the difference $g(n, r)-n^{2} / 2$ is always positive. Hence, by Theorem 2.5, $G$ is not hamiltonian. Consequently, Theorem 2.2 implies that there exists a positive integer $m \leq n / 2$ such that at least one of the sets $S_{m}=\left\{x \in X: d_{G}(x) \leq m\right\}$, $T_{m}=\left\{y \in Y: d_{G}(y) \leq m\right\}$ has cardinality greater than or equal to $m$.

Let $l$ be the least such $m$. Without loss of generality, we may assume that $l$ is realized in $X$; i.e., $\left|\left\{x \in X: d_{G}(x) \leq l\right\}\right| \geq l$. Order the vertices of $X=\left\{x_{1}, \ldots, x_{n}\right\}$ so that $r \leq d_{G}\left(x_{1}\right) \leq \cdots \leq$ $d_{G}\left(x_{n}\right)$. Then, by minimality of $l$, we have $l=\min \left\{i: d_{G}\left(x_{i}\right) \leq i\right\}$. Of course, $r \leq l \leq n / 2$. Put $L=\left\{x_{1}, \ldots, x_{l}\right\}$.

The rest of the proof proceeds in two cases, depending on $l$ being equal to or greater than $r$. 


\section{Case 1:}

$l=r$. We will first show that all the vertices of $Y$ have degrees greater than $r$. Suppose to the contrary that there exists $y_{1} \in Y$ with $d_{G}\left(y_{1}\right)=r$. Then

$$
\left\|G-\left\{x_{1}, y_{1}\right\}\right\| \geq g(n, r)-2 r=n^{2}-n-n r+r^{2}-r+1,
$$

and $\delta\left(G-\left\{x_{1}, y_{1}\right\}\right) \geq r-1$. On the other hand, by Theorem 2.3 .

$$
\left\|G-\left\{x_{1}, y_{1}\right\}\right\| \leq(n-1)(n-r)+(r-1)^{2}=n^{2}-n-n r+r^{2}-r+1 .
$$

Hence $d_{G}\left(x_{1}\right)+d_{G}\left(y_{1}\right)=2 r$ so that $x_{1} y_{1} \notin E$ and $G-\left\{x_{1}, y_{1}\right\}$ equals $G(n-1, r-1)$. By comparison of degrees, one readily verifies that $x_{1}$ belongs to that colour class of $G$ that contains $P \cup Q$ of $G(n-1, r-1)$; in fact, $L=\left\{x_{1}\right\} \cup P$. Consider the sets $R$ and $S$ of the other colour class of $G(n-1, r-1)$. As $\left|N_{G}\left(x_{1}\right)\right|=r>|R|$ and $x_{1} y_{1} \notin E$, it follows that either $x_{1}$ has neighbours in both $R$ and $S$ or else it has at least two neighbours in $S$. In any case, as in the proof of Lemma 5.1, one easily finds a cycle of length $2 n-2$ in $G$, omitting $y_{1}$ and a vertex of $P$; a contradiction. Thus $d_{G}(y) \geq r+1$ for every $y \in Y$.

Next observe that every vertex of $Y$ has a neighbour in $L$. Suppose otherwise, and let $y_{1} \in Y$ be such that $N_{G}\left(y_{1}\right) \subset X \backslash L$. Notice that all vertices of $X \backslash L$ have degrees greater than $r$, for otherwise $g(n, r) \leq\|G\| \leq(r+1) r+(n-r-1) n=g(n, r)-1$. Consequently, by removing $y_{1}$ and a vertex of $L$, say $x_{1}$, we do not decrease the minimal degree in the remainder of $G$. But, as $N_{G}\left(y_{1}\right) \subset X \backslash L$, we have $d_{G}\left(y_{1}\right) \leq n-r$, hence $d_{G}\left(x_{1}\right)+d_{G}\left(y_{1}\right) \leq r+(n-r)=n$, and by Lemma 5.1. $G$ contains a cycle of lenth $2 n-2$; a contradiction.

Consider the graph $G-L$. Notice that

$$
\|G-L\| \geq g(n, r)-r^{2}=n^{2}-n-n r+r+1 .
$$

Moreover, we claim that $d_{G-L}(x)+d_{G-L}(y) \geq n$ for every pair of non-adjacent $x \in X \backslash L$ and $y \in Y$. For if $d_{G-L}(x)+d_{G-L}(y) \leq n-1$ for a pair of non-adjacent $x \in X \backslash L$ and $y \in Y$, then, by the above inequality,

$$
\|(G-L)-\{x, y\}\| \geq n^{2}-2 n-n r+r+2>(n-r-1)(n-1),
$$

which contradicts $(G-L)-\{x, y\}$ being a bipartite graph with colour classes of cardinality $n-r-1$ and $n-1$.

Taking into account that every vertex in $Y$ has a neighbour in $L$, we now obtain that

$$
d_{G}(x)+d_{G}(y) \geq n+1 \quad \text { for all non-adjacent } y \in Y \text { and } x \in X \backslash L .
$$

Let $\widetilde{G}$ be the bipartite graph obtained from $G$ by joining all the non-adjacent vertices of $Y$ and $X \backslash L$. As $|X \backslash L|=n-r$ and every $y \in Y$ has a neighbour in $L$, we get that $d_{\widetilde{G}}(y) \geq n-r+1$ for all $y \in Y$. Hence $d_{\widetilde{G}}(x)+d_{\widetilde{G}}(y) \geq n+1$ for every pair of non-adjacent vertices $x \in X$ and $y \in Y$. Therefore, joining all the non-adjacent vertices of $X$ and $Y$ in $\widetilde{G}$ with degree sum of at least $n+1$ yields a complete bipartite graph $K_{n, n}$. As $\widetilde{G}$ was obtained from $G$ also by joining certain non-adjacent vertices of $X$ and $Y$ with degree sum of at least $n+1$, this shows that the $(n+1)$-biclosure of $G$ equals $K_{n, n}$. Thus, by Theorem 2.7 $G$ contains a Hamilton cycle, which, as we observed at the begining of this proof, is impossible. 


\section{Case 2:}

$l \geq r+1$. In this case $n \geq 2 r+2$ (as $l \leq n / 2)$ and $r \geq 2$ (for otherwise $l=r=1$, by minimality); hence $|L| \geq 3$. Moreover, $d_{G}\left(x_{l-1}\right)=d_{G}\left(x_{l}\right)=l$, by minimality of $l$.

Suppose first that $d_{G}(x)+d_{G}(y) \geq n+2$ for every pair of non-adjacent $x \in X \backslash L$ and $y \in Y$. Let $G^{\prime}$ be the bipartite graph obtained from $G$ by joining all the non-adjacent vertices of $X \backslash L$ and $Y$. We claim that every $y \in Y$ has a neighbour in $L$ (in $G^{\prime}$ ). Suppose otherwise, and let $y_{1} \in Y$ be such that $N_{G^{\prime}}\left(y_{1}\right) \subset X \backslash L$. Then $d_{G^{\prime}}\left(y_{1}\right) \leq n-l$, hence $d_{G^{\prime}}\left(x_{1}\right)+d_{G^{\prime}}\left(y_{1}\right) \leq n$. Moreover, $\delta\left(G^{\prime}-\left\{x_{1}, y_{1}\right\}\right) \geq r$, as all the vertices in $X \backslash L$ have degrees of at least $l \geq r+1$, and $d_{G^{\prime}}(y) \geq n-l \geq l \geq r+1$ for all $y \in Y$. Then Lemma 5.1 implies that $G^{\prime}$ contains a cycle of length $2 n-2$, and hence, by Theorem 2.8 . so does $G$; a contradiction.

Notice that $G^{\prime}$ was obtained from $G$ by joining only pairs of vertices with degree sum of at least $n+2$. Also, as every vertex $y \in Y$ has a neighbour in $L$ (in $G^{\prime}$ ), we have $d_{G^{\prime}}(y) \geq n-l+1$. Recall that $d_{G^{\prime}}\left(x_{l}\right)=d_{G}\left(x_{l}\right)=l$ and $d_{G^{\prime}}\left(x_{l-1}\right)=d_{G}\left(x_{l-1}\right)=l$. Hence

$$
d_{G^{\prime}}\left(x_{l}\right)+d_{G^{\prime}}(y) \geq n+1 \quad \text { and } \quad d_{G^{\prime}}\left(x_{l-1}\right)+d_{G^{\prime}}(y) \geq n+1 \quad \text { for all } y \in Y .
$$

Let $G^{(2)}$ be the graph obtained from $G^{\prime}$ by joining $x_{l}$ and $x_{l-1}$ with all the vertices of $Y$. Then $d_{G^{(2)}}(y) \geq$ $n-l+2$ for all $y \in Y$, and as $d_{G^{(2)}}\left(x_{l-2}\right)=d_{G}\left(x_{l-2}\right) \geq l-1$ (by minimality of $l$ ), we get that

$$
d_{G^{(2)}}\left(x_{l-2}\right)+d_{G^{(2)}}(y) \geq n+1 \quad \text { for all } y \in Y \text {. }
$$

Let now $G^{(3)}$ be the graph obtained from $G^{(2)}$ by joining $x_{l-2}$ with all the non-adjacent vertices of $Y$. In general, let $G^{(m)}(m \geq 3)$ be obtained from $G^{(m-1)}$ by joining $x_{l-m+1}$ with all the non-adjacent vertices of $Y$. Then $G^{(l)}=K_{n, n}$, and $G^{(m)}$ is obtained from $G^{(m-1)}$ by joining only pairs of vertices with degree sum of at least $n+1$. Thus $G^{(l)}=B C l_{n+1}(G)$, so that the $(n+1)$-biclosure of $G$ is a complete bipartite graph. Now Theorem 2.7 implies that $G$ contains a Hamilton cycle, which again leads to contradiction.

To complete the proof, it remains to consider the case when there is a pair of non-adjacent $x^{0} \in X \backslash L$ and $y^{0} \in Y$ with $d_{G}\left(x^{0}\right)+d_{G}\left(y^{0}\right) \leq n+1$. This however can only happen when $n=2 r+2$ or $n=2 r+3$. For let us suppose that $n \geq 2 r+4$, and put $f(l)=l^{2}+(n-l-1)(n-1)+n+2$. We show $\|G\|<f(l)$ and $f(l) \leq g(n, r)$, and thus obtain a contradiction with the assumption $\|G\| \geq g(n, r)$. If $G$ contains a pair of non-adjacent vertices $x \in X \backslash L$ and $y \in Y$ with $d_{G}(x)+d_{G}(y) \leq n+1$, then

$$
\|G\| \leq|L| \cdot l+|X \backslash(L \cup\{x\})| \cdot|Y \backslash\{y\}|+d_{G}(x)+d_{G}(y) \leq f(l)-1 .
$$

As the derivative of $f$ equals $f^{\prime}(l)=-n+2 l+1$, it follows that $f(l)$ is decreasing for $l \leq(n-1) / 2$, and hence maximal at $l=r+1$. One immediately verifies that $f(r+1) \leq g(n, r)$ for $n \geq 2 r+4$. If, on the other hand, $l>(n-1) / 2$, then $l=n / 2$ (since $l \leq n / 2)$, and it is again immediate to check that $f(n / 2) \leq g(n, r)$ for $n \geq 2 r+4$.

\section{Subcase 2.1:}

$n=2 r+2$. Then $r+1 \leq l \leq n / 2$ yields $l=r+1$, and we obtain

$$
\left\|G-\left\{x^{0}, y^{0}\right\}\right\| \geq g(2 r+2, r)-(2 r+3)=3 r^{2}+3 r .
$$


On the other hand,

$$
\left\|G-\left\{x^{0}, y^{0}\right\}\right\| \leq|L| \cdot l+\left|X \backslash\left(L \cup\left\{x^{0}\right\}\right)\right| \cdot\left|Y \backslash\left\{y^{0}\right\}\right|=3 r^{2}+3 r+1 .
$$

Hence

$$
3 r^{2}+3 r \leq\left\|G-\left\{x^{0}, y^{0}\right\}\right\| \leq 3 r^{2}+3 r+1 \text { and } 2 r+2 \leq d_{G}\left(x^{0}\right)+d_{G}\left(y^{0}\right) \leq 2 r+3 .
$$

Suppose first that $\left\|G-\left\{x^{0}, y^{0}\right\}\right\|=3 r^{2}+3 r+1$. Then, by (2), $d_{G}(x)=l$ for all $x \in L$, and $N_{G}\left(y^{0}\right) \cap L=\emptyset$; in particular, $d_{G}\left(x_{1}\right)+d_{G}\left(y^{0}\right) \leq l+(n-l)=n$. Moreover, $N_{G}(y) \supset X \backslash\left(L \cup\left\{x^{0}\right\}\right)$ for all $y \in Y \backslash\left\{y^{0}\right\}$, and $d_{G}(x) \geq r+1$ for all $x \in X$, so that $\delta\left(G-\left\{x_{1}, y^{0}\right\}\right) \geq r$, and by Lemma 5.1. $G$ contains a cycle of length $2 n-2$; a contradiction.

Therefore we may assume that $\left\|G-\left\{x^{0}, y^{0}\right\}\right\|=3 r^{2}+3 r$. By $(1), d_{G}\left(x^{0}\right)+d_{G}\left(y^{0}\right)=2 r+3$, and what's more, $d_{G}(x)+d_{G}(y) \geq 2 r+3$ for all non-adjacent $x \in X \backslash L$ and $y \in Y$. Indeed, if $d_{G}\left(x^{1}\right)+d_{G}\left(y^{1}\right) \leq 2 r+2$ for some non-adjacent $x^{1} \in X \backslash L$ and $y^{1} \in Y$, then by (1) and (2), $\left\|G-\left\{x^{1}, y^{1}\right\}\right\|=3 r^{2}+3 r+1$, which leads to contradiction, as above.

We will now show that $\left|N_{G}(L)\right|>r+1$. Suppose otherwise, that is, suppose $\left|N_{G}(L)\right|=l=r+1$. Then $N_{G}\left(y^{0}\right) \cap L=\emptyset$, for else $N_{G}(L) \ni y^{0}$ implies

$$
\left\|G-\left\{x^{0}, y^{0}\right\}\right\| \leq|L| \cdot(l-1)+\left|X \backslash\left(L \cup\left\{x^{0}\right\}\right)\right| \cdot\left|Y \backslash\left\{y^{0}\right\}\right|=3 r^{2}+2 r,
$$

which is impossible. Therefore $d_{G}\left(y^{0}\right)=n-l-1=r$; in particular, $d_{G}\left(x_{1}\right)+d_{G}\left(y^{0}\right) \leq l+r<n$. Notice that, as $G-\left\{x^{0}, y^{0}\right\}$ only has one edge less than the right-hand side of (2), every neighbour of $y^{0}$ in $G$ has degree at least $n-2=2 r$, and every neighbour of $x_{1}$ has at least $l-1=r$ other neighbours in $L$ ( $x_{1}$ being the only vertex whose degree could be less than $l$ ). Thus $\delta\left(G-\left\{x_{1}, y^{0}\right\}\right) \geq r$, and we get a contradiction, by Lemma 5.1. Thus $\left|N_{G}(L)\right|>r+1$.

It is now not difficult to see that $B C l_{n+1}(G)=K_{n, n}$ : Recall that we have verified that $d_{G}(x)+$ $d_{G}(y) \geq 2 r+3=n+1$ for all non-adjacent $x \in X \backslash L$ and $y \in Y$. Let $G^{\prime}$ be the graph obtained from $G$ by joining all the non-adjacent vertices of $X \backslash L$ and $Y$. Next observe that, by minimality of $l=r+1, d_{G^{\prime}}\left(x_{r+1}\right)=d_{G}\left(x_{r+1}\right)=r+1$, and as $\left|N_{G}(L)\right|>r+1$, at least one non-neighbour of $x_{r+1}$, say $y^{\prime}$, has a neighbour among the other vertices of $L$. Hence $\left|N_{G^{\prime}}\left(y^{\prime}\right)\right| \geq|X \backslash L|+1$, so that $d_{G^{\prime}}\left(x_{r+1}\right)+d_{G^{\prime}}\left(y^{\prime}\right) \geq(r+1)+(r+2)=n+1$. Let $G^{(2)}$ be obtained from $G^{\prime}$ by joining $x_{r+1}$ with $y^{\prime}$, and hence increasing the degree of $x_{r+1}$ to $r+2$. Then $d_{G^{(2)}}\left(x_{r+1}\right)+d_{G^{(2)}}(y) \geq n+1$ for all $y \in Y$. Let $G^{(3)}$ be obtained from $G^{(2)}$ by joining $x_{r+1}$ with all the non-adjacent vertices of $Y$. Now $d_{G^{(3)}}(y) \geq r+2$ for all $y \in Y$. By minimality of $l$ again, $d_{G^{(3)}}\left(x_{r}\right)=d_{G}\left(x_{r}\right)=r+1$, and hence $d_{G^{(3)}}\left(x_{r}\right)+d_{G^{(3)}}(y) \geq 2 r+3$ for all $y \in Y$. Let $G^{(4)}$ be obtained from $G^{(3)}$ by joining $x_{r}$ with all the non-adjacent vertices of $Y$. Then $d_{G^{(4)}}(y) \geq r+3$ for all $y \in Y$, and hence, as $\delta\left(G^{(4)}\right) \geq \delta(G) \geq r$, $d_{G^{(4)}}(x)+d_{G^{(4)}}(y) \geq 2 r+3$ for all non-adjacent $x \in X$ and $y \in Y$. Joining all the non-adjacent pairs $x \in X, y \in Y$ of $G^{(4)}$ with degree sum of at least $n+1$ we thus obtain $K_{n, n}$. Since at each stage we only joined pairs of vertices with degree sum of at least $n+1$, this shows that $K_{n, n}=B C l_{n+1}(G)$. By Theorem 2.7. $G$ contains a Hamilton cycle; a contradiction. 


\section{Subcase 2.2:}

$n=2 r+3$. Again, $r+1 \leq l \leq n / 2$ yields $l=r+1$, and we have

$$
\left\|G-\left\{x^{0}, y^{0}\right\}\right\| \geq g(2 r+3, r)-(2 r+4)=3 r^{2}+6 r+3,
$$

and, on the other hand,

$$
\left\|G-\left\{x^{0}, y^{0}\right\}\right\| \leq|L| \cdot l+\left|X \backslash\left(L \cup\left\{x^{0}\right\}\right)\right| \cdot\left|Y \backslash\left\{y^{0}\right\}\right|=3 r^{2}+6 r+3 .
$$

Therefore both inequalities must, in fact, be equalities; in particular, $d_{G}\left(x_{1}\right)=l$ and $d_{G}(x) \geq r+1$ for all $x \in X, N_{G}\left(y^{0}\right) \cap L=\emptyset$, so that $d_{G}\left(y^{0}\right) \leq n-l$, and finally $\left|N_{G}(y)\right| \geq\left|X \backslash\left(L \cup\left\{x^{0}\right\}\right)\right|=r+1$ for all $y \in Y \backslash\left\{y^{0}\right\}$. Thus, again, $G$ with the vertices $x_{1}, y^{0}$ satisfies the assumptions of Lemma 5.1. hence $G$ contains a cycle of length $2 n-2$; a contradiction. This completes the proof of Theorem B.

\section{Acknowledgements}

We are grateful to Evelyne Flandrin for her valuable comments and numerous discussions regarding the problems of this paper. We would also like to thank Artur Fortuna for an inspiring conversation. 
Ore and Erdös type conditions for long cycles in balanced bipartite graphs

\section{References}

[1] J. Adamus, A note on a degree sum condition for long cycles in graphs, preprint arXiv:0711.4394v2.

[2] J. Adamus, On the cycle structure of hamiltonian $\delta$-regular bipartite graphs of order $4 \delta$, preprint arXiv:0711.4426v2.

[3] L. Adamus, Edge condition for long cycles in bipartite graphs, Discrete Math. Theor. Comput. Sci. 11:2 (2009), 25-32.

[4] D. Amar, O. Favaron, P. Mago and O. Ordaz, Biclosure and bistability in a balanced bipartite graph, J. Graph Theory 20 (1995), 513-529.

[5] K.S. Bagga and B.N. Varma, Hamiltonian properties in bipartite graphs, Bull. Inst. Combin. Appl. 26 (1999), 71-85.

[6] C. Balbuena, P. García-Vázquez, X. Marcote and J.C. Valenzuela, Counterexample to a conjecture of Györi on $C_{2 l}$-free bipartite graphs, Discrete Math. 307 (2007), 748-749.

[7] J. A. Bondy and V. Chvátal, A method in graph theory, Discrete Math. 15 (1976), 111-135.

[8] R.C. Entringer and E.F. Schmeichel, Edge conditions and cycle structure in bipartite graphs, Ars Combinatoria 26 (1988), 229-232.

[9] P. Erdős, Remarks on a paper of Pósa, Magyar Tud. Akad. Mat. Kutató Int. Kőzl. 7 (1962) 227-229.

[10] N. Linial, A lower bound for the circumference of a graph, Discrete Math. 15 (1976), 297-300.

[11] J. Moon and L. Moser, On hamiltonian bipartite graphs, Israel J. Math. 1 (1963), 163-165.

[12] O. Ore, Note on Hamilton circuits, Amer. Math. Monthly 67 (1960), 55.

[13] A.P. Wojda and M. Woźniak, Orientations of hamiltonian cycles in bipartite digraphs, Period. Math. Hungar. 28 (1994), 103-108.

[14] D.R. Woodall, Sufficient conditions for circuits in graphs, Proc. London Math. Soc. (3) 24 (1972), 739-755. 
